Revista

Multi-Ensayos

Vol. 5, $\mathrm{N}^{\circ} 10$

ISSN: 2412-3285

https://multiensayos.unan.edu.ni

DOI: https://doi.org/10.5377/multiensayos.v5i10.8871

\title{
Experiencia docente en la asignatura Teorías de las Relaciones Internacionales
}

\section{Teaching experience in the Theories of International Relations course}

\author{
Junieth Osegueda Herrera ${ }^{1}$
}

Recibido: 22 de mayo de 2019. Aceptado: 20 de junio de 2019

\section{RESUMEN}

La finalidad del presente ensayo es compartir mi experiencia docente al impartir la asignatura Teorías de las relaciones internacionales a los estudiantes del segundo año de la carrera Ciencia Política y Relaciones Internacionales. Se analiza la importancia de esta asignatura en la Carrera, tomando en consideración las concepciones teóricas que fundamentan su objeto de estudio, los principales problemas mundiales y los actores internacionales. Así mismo, se reflexiona como esta asignatura conduce a los futuros politólogos y relacionistas internacionales e interesados en los problemas que acontecen en el escenario social Internacional, tener una visión clara de las principales teorías y enfoques holístico de las relaciones Internacionales.

Palabras claves: Teorías; Relaciones Internacionales; estrategias.

\section{ABSTRACT}

The purpose of this essay is to share my teaching experience during the development of the course of International Relations Theories, to students from the second year of the major Political Sciences and International Relationships. The importance of this course in this major is analyzed, taking into consideration the theoretical conceptions that are based on the object of study, main global problems, and the international actors. Similarly, it is a reflection on how this course could lead to future international political and relations academics to get interested in the problems that happen in the international social scene, to have a clear vision of the main theories and holistic approaches of International Relations.

Keywords: Theories; International Relations; strategies.

1 Docente UNAN-Managua/FAREM-Estelí. Correo electrónico: juniethosegueda@gmail.com. (c) 2019 Revista Multi-Ensayos. 


\section{INTRODUCCIÓN}

Este ensayo tiene como objetivo compartir mi experiencia docente durante el desarrollo de la asignatura Teoría de las Relaciones Internacionales, la cual ha sido satisfactoria y ha significado un gran compromiso, ya que es una asignatura orientada a la formación de los nuevos teóricos y especialistas del campo internacional, en su hacer y en el alcance de los objetivos propuestos en el programa de evaluación, remarcando la contextualización de los aprendizajes. Brindando a los estudiantes la capacidad de poder interpretar, estudiar y situar las principales corrientes interpretativas de las relaciones internacionales, comprender la dimensión teórica de las mismas, su alcance, las fuerzas que las estructuran, los fines y objetivos que movilizan a los diferentes actores del escenario internacional para la coexistencia, autodestrucción de las partes en contienda y conflicto y en contra posiciones antagónicas por la supervivencia y existencia de la humanidad. Asimismo, otorga a las y los estudiantes las facultades, habilidades y destrezas en el análisis de los contextos internacionales.

La teoría de las relaciones internacionales es una ciencia nueva, en palabras del historiador estadounidense Bruce Hoffmann (1954), se define como: "Estudio sistemático de fenómenos observables que intenta descubrir las variables principales, explicar el comportamiento y revelar los tipos característicos de relaciones entre unidades internacionales".

También es importante referir que siguiendo al maestro Celestino Del Arenal, se entiende por relaciones internacionales:

"El conjunto de relaciones sociales que configuran la sociedad internacional, tanto las de carácter político como las no políticas, sean económicas, culturales, humanitarias, religiosas, etc., tanto las que se producen entre los Estados como las que tienen lugar entre otros actores de la sociedad internacional y entre estos y los Estados. De esta forma puede decirse que las relaciones internacionales es la ciencia que se ocupa de la sociedad internacional". ARENAL, Celestino del (2007)

La teoría de las relaciones internacionales gira en torno a cuatro debates, entendidos como una definición de características y temáticas fundamentales en orden cronológico. Los términos: enfoques, paradigmas, modelos teóricos, escuelas, etc. también pueden utilizarse para referirse a los mismos. El primer debate alude al Realismo versus idealismo (1919-1949), el segundo debate: Ciencias versus humanidades (19491979), el tercer debate: Globalidad versus estatocentrismo (1979-1989) y el cuarto debate: Anarquía-caos, homogeneidad-heterogeneidad, inclusión-exclusión-actualidad (1989).

Al desarrollar esta asignatura se logró vincular la teoría con la práctica, permitiendo a las y los estudiantes la oportunidad de conocer los campos de acción en donde intervendrán profesionalmente. Por ejemplo, como analista de la política exterior nicaragüense (representando al País ante organismos, misiones diplomáticas y consulares) y asesor en negociación de Tratados o Acuerdos Internacionales. En ambos casos, las atribuciones principales son: comprender y explicar el funcionamiento de las relaciones Internacionales, capacidad de negociación y persuasión en la solución y manejo de conflictos internacionales, visualiza la opción y posición nacional dentro de los diferentes escenarios internacionales, se compromete con la sociedad y respeta los derechos humanos. 
Esta asignatura es muy importante en la Carrera Ciencia Política y Relaciones Internacionales ya que las teorías que fundamentan el estudio de las mismas, enfocan su debate en los paradigmas, debido a la propia evolución y cambio de la realidad internacional que es objeto de estudio, a los profundos y radicales cambios sociales, políticos, económicos y científico- técnicos, al aumento cuantitativo en el escenario internacional de los actores y de las interacciones entre ellos, a la creciente influencia de los movimientos sociales y políticos en las relaciones internacionales, a la heterogeneidad del sistema internacional a partir de la revolución bolchevique, a los horrores de la Gran Guerra (primera guerra mundial) a los deseos de instaurar un orden de paz y seguridad, a la globalización caracterizada por las revoluciones tecnológicas e industriales, por el capitalismo salvaje y el fenómeno de la creciente interdependencia simétrica y asimétrica, a la aparición de nuevos y dramáticos problemas mundiales como: la desigualdad social, la pobreza, crisis energética, escasez de agua, el petróleo, el cambio climático, la corrupción, la hegemonización del sistema dominante, las guerras, el terrorismo etc. que han experimentado las relaciones internacionales y con ellas el sistema internacional y que han influido potentemente en la evolución de las propias concepciones científicas de las relaciones internacionales y de los paradigmas en los cuales se fundamentan las mismas.

\section{DESARROLLO}

Durante el desarrollo de esta asignatura, se utilizó el enfoque constructivista humanista, para promover la actividad y participación de los y las estudiantes como sujetos activos del proceso pedagógico y su vinculación con la realidad nacional e internacional, su problemática y las alternativas de solución. Así mismo, se utilizó metodologías participativas dentro del aula de clase y se procuró fomentar el contacto de las y los estudiantes con la realidad internacional, preparándolos para su futura incursión en el campo de acción del teórico de las relaciones internacionales a través de:

\section{Estrategias desde la facilitación docente}

1. Exposiciones teóricas de cada contenido, generando lluvia de ideas durante el desarrollo de cada clase, para crear un diagnostico acerca de las principales líneas filosóficas y doctrinarias que conllevaron a establecer las bases teóricas de las relaciones internacionales (Tucidides, Herodoto, Hobbes, Maquiavelo, Rousseau, Kant, Hugo Groccio, Kautilya, Parson),

2. Diagrama de Ciclos que se les presentó a las y los estudiantes para que apreciaran cada uno de los métodos coercitivos (Embargos, represalias, maniobras militares, retiros de misiones, ruptura de relaciones, interrupción de comunicaciones, boicots e Intervenciones) adoptados en el transcurso de las relaciones internacionales por los actores del sistema internacional, tomando en consideración la aplicación práctica del paradigma tradicionalista.

3. Presentación gráfica de las relaciones entre Estados, grupos financieros, empresas, compañías y corporaciones transnacionales que operan en la Región Centroamericana y Nicaragua. En este caso, los alumnos indagaron acerca de las interacciones entre otros Estados como China, Corea del Sur, Federación Rusa, Unión Europea y Empresas como Walmart, Unilever, Claro, entre otras, con Centroamérica y particularmente con Nicaragua. 


\section{Aprendizaje colaborativo desde los estudiantes}

1. Actividades grupales diseñadas para la valoración de los contenidos estudiados, con clases prácticas donde se priorizó el análisis crítico, reflexivo y propositivo con el fin de potencializar en las y los estudiantes capacidades y habilidades necesarias en el campo de su formación profesional.

En este caso, los alumnos se organizaron en equipos de seis integrantes e indagaron acerca de la política exterior de Estados Unidos, según su grado de poder, área de influencia, madures política, dirigida a Latinoamérica con la Doctrina de Monroe a partir de 1823 y posteriormente con la Diplomacia del dólar en 1909. Refiriendo una alumna que: ambas políticas tenían como objetivo expandir su dominio principalmente político y económico en nuestra Región latinoamericana. El análisis de estas temáticas se les orientó según la concepción marxista de la realidad internacional. Siguiendo esta misma estrategia, los alumnos también investigaron sobre la intervención norteamericana en Nicaragua en 1912, que según un alumno representante de grupo: "fue el hecho que marcó la historia de Nicaragua con la sangrienta guerra nacional y el continuo injerencismo estadounidense en nuestro País hasta nuestros días".

2. Cuestionarios de interrogantes filosóficas, políticas, histórico-jurídicas, socio- políticas que se les dejó al final de cada clase, lo cual contribuyó a que las y los estudiantes puedan responder aspectos teóricos de las relaciones internacionales

3. Dos debates sobre los principales problemas de teorización internacional. En esta actividad se les orientó a los alumnos dividirse en dos grupos para adoptar un determinado enfoque en la comprensión internacional. En el primer debate, un grupo representó al cientifismo y el otro representó al tradicionalismo. En el segundo debate, un grupo representó al idealismo filosófico y el otro al realismo político. Refiriendo una alumna representante de grupo que: "esta actividad les encantó porque les permitió poner en práctica el análisis crítico-reflexivo necesario para la comprensión de los referidos enfoques teóricos".

4. Círculos analíticos e interpretativos sobre hechos de carácter nacional o internacional por medio de ejemplos prácticos e históricos en el marco de las principales doctrinas teóricas de las líneas del pensamiento mundial, en cuyo caso las y los estudiantes analizaron la interdependencia entre la Federación Rusa y La Unión Europea, entre Estados Unidos y la República Popular de China, entre Estados Unidos y los Países Centroamericanos.

En esta actividad los alumnos analizaron si las interdependencias entre estas Naciones realmente son simétricas o asimétricas, manifestando un alumno representante de grupo que "en los dos primeros casos es simétrica porque los beneficios y costes son recíprocos para ambas partes pero que el último caso se trata de una interdependencia asimétrica porque constituye una relación de pura dependencia económica de nuestros Países centroamericanos respecto a Estados Unidos que es su principal inversor y acreedor".

Al respecto, reforcé sus aseveraciones explicándoles que Estados Unidos al ser la primera potencia económica y militar a nivel mundial y aprovechado su posición como uno de los cinco miembros 
permanentes del Consejo de seguridad de la Organización de Naciones Unidas impone a nuestras Naciones Centroamericanas subdesarrolladas, sus políticas neoliberales (imperialistas) que erosionan cada día nuestra soberanía nacional, la autodeterminación de nuestros pueblos, la defensa de nuestra economía nacional y que violentan nuestros derechos humanos y libertad ideológica puesto que su prioridad es satisfacer sus propios intereses económicos aprovechando nuestra posición geográfica estratégica para el comercio internacional, nuestro territorio para establecer a bajos costos sus grandes transnacionales, nuestros recursos naturales para explotarlos, nuestras materias primas para comercializarlas y nuestra mano de obra barata.

Por lo cual se les instó a las y los estudiantes a mantener vivo el sentimiento Patriótico, revolucionario, a rescatar nuestra identidad nacional y a mantenernos unidos como hermanos solidarios en defensa de nuestra querida Nicaragua dentro del marco del respeto, la justicia y la paz.

5. Simulación y juegos de roles, en donde cada estudiante protagonizó a un diplomático en el ejercicio de sus funciones. En este caso, se organizaron para recrear la adopción de la Agenda 20/30 por parte de la Asamblea general de las Naciones Unidas. "Esta actividad fue una de las más creativas y enriquecedoras para nosotros ya que logramos apropiarnos de los diecisiete objetivos de aplicación universal para lograr el desarrollo sostenible en todos los Países en el año dos mil treinta" manifestó una estudiante, en representación de su grupo.

6. Elaboración de guías de cuestionamiento referente a los distintos planteamientos expuestos o abordados en cada unidad, actividad en la cual los alumnos propiciaron el razonamiento lógico y la expresividad sólida, fluida y coherente a cada una de las afirmaciones o cuestionamientos que le fueron presentados.

\section{Analítico-reflexivo}

1. Informe analítico-reflexivo sobres las leyes: 800 (Ley del régimen jurídico de el gran canal interoceánico de Nicaragua y crea la autoridad de el gran canal interoceánico de Nicaragua) y 840 (Ley especial para el desarrollo de infraestructura y transporte nicaragüense atingente a el canal, zonas de libre comercio e infraestructuras asociadas). En esta actividad las y los estudiantes analizaron las referidas leyes tomando en consideración la axiología de los modelos alternativos incluyendo el de la soberanía nacional y la aplicación práctica del paradigma de la Dependencia.

2. Cuadro comparativo de análisis individual, referente al análisis entre el Dr-CAFTA y el ALBA, en el cual determinaron las desventajas del Dr-CAFTA y las ventajas del ALBA para la Región Centroamericana.

\section{Redacción, análisis y pensamiento crítico}

1. Redacción de dos ensayos, que se les orientó presentar de manera individual, uno referente a su apreciación personal y objetiva sobre las diversas corrientes teóricas en el aprendizaje de las nuevas realidades internacionales en el plano mundial, nacional, local y comunitario, y el otro, referente a su 
valoración de la importancia que tienen las causas en favor de la Justicia, la Igualdad, la Cooperación entre los pueblos del mundo fortaleciendo los valores de la diversidad, el respeto y la tolerancia. Algunos alumnos trabajaron su ensayo, apoyándose en documentos relativos a los organismos especializados de la ONU (Organización de Naciones Unidas), otros se apoyaron en documentos relativos al ALBA (Alianza Bolivariana para los Pueblos de nuestra América).

2. Elaboración de tres proyectos en grupos de seis integrantes. El primero, sobre organización internacional que responda a los factores de cambios de los escenarios internacionales y a la solución de los graves y puntuales problemas del mundo en sustitución del actual sistema de organización mundial. El segundo, sobre integración latinoamericana en base a la teoría funcionalista, en cuyo caso tomaron como referencia los aspectos políticos, económicos y culturales de la región; y el tercero, sobre negociación internacional con intervención de terceros respecto al conflicto entre Nicaragua y Colombia, en base a la teoría de la negociación.

Es oportuno mencionar, que las y los estudiantes también debatieron acerca de los hechos, eventos, situaciones, actos, realidades y fenómenos internacionales históricos y actuales en los que se manifiesten las diferentes doctrinas teóricas de las relaciones internacionales, tal es el caso de las guerras en Medio Oriente, el injerencismo estadounidense en la República Bolivariana Venezuela, el histórico bloqueo económico a Cuba, la tensión nuclear entre los Estados Asiáticos: India y Pakistán, las consecuencias de la llamada guerra del Pacífico suscitada entre las Repúblicas latinoamericanas: Chile, Bolivia y Perú, la actual tensión entre Corea del Norte y Estados Unidos, identificando en cada caso los elementos teóricos, las variables conceptuales dominantes y propias de cada tendencia e interpretación teórica de la realidad internacional, el actual sistema internacional (si es unipolar, bipolar o multipolar) así como diferenciando los principales patrones en los diversos enfoques teóricos en el estudio de las relaciones internacionales.

Por último, es importante destacar que la Universidad Nacional Autónoma de Nicaragua, (UNAN-Managua) como institución de Educación Superior y de carácter público, asume su compromiso y responsabilidad social de formar profesionales integrales, por medio de un proceso de enseñanza aprendizaje de calidad. En mi caso particular me he apropiado de ese proceso el cual "tiene como base un modelo pedagógico que centra su atención en el estudiante, que asume su rol activo y participativo con una alta responsabilidad en el desarrollo de su aprendizaje autónomo y estratégico" Modelo educativo, 2011, p.23) cuyo papel primordial nos corresponde a nosotros como docentes ya que somos los responsables de facilitar y hacer efectivo ese proceso de enseñanza aprendizaje de calidad.

\section{CONCLUSIÓN}

La asignatura Teoría de las Relaciones Internacionales favoreció en las y los estudiantes el desarrollo de habilidades, facultades, destrezas en el análisis de los contextos internacionales, valores, actitudes humanas acordes con el perfil profesional del relacionista internacional y competencias laborales específicas como: el saber relacionar los sucesos internacionales con las teorías formuladas por los principales autores de las Relaciones Internacionales, la proactividad, el compromiso, la creatividad e iniciativa-innovación, sobre todo para la resolución de conflictos. 
Se logró vincular la teoría con la práctica, permitiéndoles a las y los estudiantes la oportunidad de conocer los campos de acción en donde intervendrán profesionalmente. Al respecto, se les explicó el perfil del politólogo y relacionista internacional haciendo énfasis en cargos como: analista de la política exterior nicaragüense (representando al País ante organismos, misiones diplomáticas y consulares) y asesor en negociación de Tratados o Acuerdos Internacionales. Por lo cual las y los estudiantes recrearon situaciones relativas a las responsabilidades antes mencionadas, asumiendo de manera individual un rol como diplomático u Asesor en negociación.

Finalmente, como docente se debe facilitar enseñanzas apegadas al modelo educativo de la UNANMANAGUA, FAREM-ESTELÍ para hacer efectiva la educación de calidad, fomentando en las y los estudiantes: La práctica de valores, la motivación, el deseo de superación personal, el estudio independiente, el trabajo en equipo, el análisis, pensamiento crítico, la reflexión; y la investigación científica.

\section{REFERENCIAS}

ARENAL, Celestino del (2007). Introducción a las Relaciones Internacionales. Madrid, Tecnos (4a edición). Reimpresión 1993. Obtenido de: http://ucm.academia.edu/CelestinodelArenal

Barbé, Esther (2007): Relaciones Internacionales, Madrid, Tecnos, $3^{a}$ ed., Obtenido de https://www.upf. edu/organitzacio/treballar/pdi/funcionari/acces_09/.../Barbe1.doc

Carta de las Naciones Unidas (San Francisco, 26 de junio de 1945). Obtenido de: https://www.oas. org/36ag/espanol/doc_referencia/Carta_NU.pdf 